\section{Silicone intraocular lens compression and double lens implants in diseased eyes}

\begin{abstract}
Purpose To assess the outcomes of double lens implants in hyperoptic eyes with associated pathology.

Method Double lens implants were used in 4 eyes of 4 patients each with a different ophthalmic or neuro-ophthalmic disease. Biometry was performed in the standard contact fashion and lens power formulae used included SRK/T, Holladay and Hoffer $Q$. Results Average spherical equivalent refraction improved from +6.875 $\mathrm{D}$ to $+0.38 \mathrm{D}$. Absolute average prediction error was greatest for SRK/T (2.65 D) and least for Holladay (1.73 D). Refractive surprises were influenced by the underlying disease process. One patient showed central lens compression.

Conclusion Underlying disease can produce biometry errors. Structural ophthalmic or neurological disease is not a contraindication to the use of double lens implants. Double lens implants are useful to correct refractive error in the presence of underlying disease.
\end{abstract}

Key words Anterior chamber, Anterior chamber depth, Best spectacle corrected visual acuity, Computerised video keratometry, Double lens implant, Intraocular lens, Phototherapeutic keratectomy

\footnotetext{
A.J. Maloof

R. Jain

M. Restori

J.D. Stevens

Departmernt of Cornea and

Refractive Surgery

Moorfields Eye Hospital

London, UK
}

Anthony J. Maloof Moorfields Eye Hospital 162 City Road

London EC1V 2PD, UK

Tel: $+44(0) 2072533411$

Fax: $+44(0) 2072534696$

e-mail: amaloof@one.net.au

Received: 21 December 1999 Accepted without revision: 14 April 2000
ANTHONY J. MALOOF, RAJNI JAIN, MARIE RESTORI, JULIAN D. STEVENS

results for each of these. Each patient suffered from a separate disease process, including Fuchs' corneal dystrophy, Grayson-Wilbrandt corneal dystrophy, vitelliform dystrophy and congenital nystagmus. From the outcomes we have tried to identify any biometry problems associated with DLIs in these diseases, and suggest appropriate solutions.

\section{Methods}

Pre-operatively, patients were assessed by an ophthalmologist. Measurements taken included keratometry, computerised video keratometry (CVK), ultrasound anterior chamber (AC) depth and contact ultrasound biometry. Pre-operative biometry findings are presented in Table 2 . Post-operatively, refraction was recorded where possible. Indications for surgery were either refractive (patient 1) or cataract (remainder).

Surgery was performed by two surgeons (J.D.S., A.J.M.). In 3 cases (patients 1, 3, 4), Chiron C11UB silicone foldable IOL, A constant 119.0, ACD Const $5.55 \mathrm{~mm}$, SF $1.79 \mathrm{~mm}$, biconvex (equiconvex) lenses, were implanted. In the fourth case (patient 2), L141U Chiron PMMA rigid lenses, A constant 117.5, ACD Const $4.67 \mathrm{~mm}$, SF $0.94 \mathrm{~mm}$ were implanted. All lenses were implanted in the bag with haptics parallel. ${ }^{7}$ Personalised lens constants were not used as an inadequate number of patients of similar axial length were available to make the results meaningful. ${ }^{8}$ Theoretical formulae utilised were either the Holladay, ${ }^{9} \mathrm{SRK} / \mathrm{T}^{10}$ or Hoffer Q. Holladay 2 software was not utilised as it was not available at the time of surgery.

The total power of the lens combinations implanted was the same as or greater than that predicted by the lens formulae.

Patients were followed up at weeks 3 and 6 and month 6 . The refractive results of the theoretical formulae are presented in Table 2. Patient 2 was not followed up at month 6 as she lived in another country and departed soon after her 3 week follow-up. 
Table 1. Pre-operative findings, including age and visual acuity

\begin{tabular}{ccccccc}
\hline Patient no. & Age (years) & Sex & Eye & BSCVA & Pathology & Biometry problem \\
\hline 1 & 53 & M & Left & $6 / 9$ & Grayson-Wilbrandt dystrophy & Keratometry, estimated corneal power \\
2 & 27 & F & Left & CF & $\begin{array}{c}\text { Nanophthalmos, glaucoma, } \\
\text { nystagmus, mature cataract, amblyopia }\end{array}$ & Keratometry, axial length (nystagmus) \\
3 & 88 & F & Left & $6 / 18$ & Vitelliform dystrophy & Axial length (maculopathy) \\
4 & 89 & F & Right & $6 / 24$ & Fuchs' corneal dystrophy & Keratometry, estimated corneal power \\
\hline
\end{tabular}

Patient 3 had an axial length biometry discrepancy noted pre-operatively, with a $1 \mathrm{~mm}$ observer-dependent variability in axial length. The reading of the most experienced ultrasonographer was utilised.

Table 2. Pre-operative biometry findings

\begin{tabular}{ccccccccc}
\hline Patient no. & Pre-operative therapy & K1 & K2 & AXL & US ACD & SRK/T & Holladay & Hoffer Q \\
\hline 1 & $+9.5 /-0.75 \times 170$ & 44.23 & 43.37 & 19.4 & - & 36.35 & 37.22 & 38.35 \\
2 & Nil & 49.78 & 49.41 & 16.8 & - & 40.28 & 40.95 & 46.17 \\
3 & 8.5 & 47.5 & 44.75 & 19.27 & 2.2 & 34.76 & 35.27 & 36.48 \\
4 & $+4.0 /-2.0 \times 100$ & 44 & 41.5 & 20.89 & 2.06 & 31.13 & 31.76 & 32.33 \\
\hline
\end{tabular}

The average pre-operative spherical equivalent was $6.875 \mathrm{D}$.

\section{Results}

Results are given in Tables 3-5. All patients experienced an improvement in uncorrected visual acuity as well as reporting a subjective improvement in vision. Best spectacle corrected visual acuity (BSCVA) was the same or better post-operatively, depending on whether surgery was for cataract or refractive reasons. No patient suffered deterioration of vision, and no patient underwent lens exchange. In all patients, capsular fibrosis was symmetrical, the haptics remained aligned and no displacement of lens optics was detected.

All formulae showed a relatively high prediction error for all patients except for the patient with Fuchs corneal dystrophy. Table 3 shows the lenses used and the postoperative refraction with BSCVA. The average preoperative refraction of 3 patients was $+6.875 \mathrm{D}$ (patient 2 was not prescribed spectacles due to very poor vision from amblyopia and cataract). The average postoperative spherical equivalent refraction was $+0.38 \mathrm{D}$, whilst the absolute average post-operative spherical equivalent was 1.63 D. (The absolute average differs from the average as opposite signs will not cancel each other out, and is therefore more meaningful.) The range of post-operative spherical equivalent refraction was $4.13 \mathrm{D}$. The predicted versus actual refraction at 6 weeks for each patient is shown in Table 4 , and the prediction error for the lens powers used in each patient is shown in Table 5. The absolute average prediction error was greatest for SRK/T (= 2.65 D), then Hoffer Q (= 1.94 D) and least for Holladay $(=1.73 \mathrm{D})$.

\section{Discussion}

Although in the bag placement of multiple lenses has declined in popularity due to interlenticular opacification, other combinations of double lens implants are still appropriate, such as placement of sulcus fixation of the anterior intraocular lens. The refractive correction of short eyes with double lens implants is very complex and must address important issues including expectation of patients, pre-operative errors in biometry, ${ }^{11}$ errors in AC depth prediction, variability of biometry measurement and the usage and interpretation of personalised constants. The achievement of emmetropia in short eyes, especially those less than $21 \mathrm{~mm}$ long, is very difficult, as every millimetre of error is associated with a greater dioptric difference than for emmetropic or myopic eyes (Fig. 1). Whilst our concerns were also directed to the refractive result, our primary goal was an improvement in visual function, and as this was achieved in all patients, no patient underwent further surgery. The diseased eyes in this series presented a challenge in biometry assessment, as well as a technical challenge intra-operatively. The problems encountered included keratometry difficulties in the presence of corneal lesions, pre-operative assessment in the presence of nystagmus, ultrasound biometry in the presence of macular disease and potential optical problems directly relating to DLIs in diseased eyes. We did not find any difficulties in obtaining pre-operative ultrasonic AC depth measurements in these eyes. Due to the variation in pathology, it is impossible to deduce statistical relevance from such a small single group. It is also

Table 3. Post-operative findings

\begin{tabular}{ccccr}
\hline Patient & Posterior IOL & Anterior IOL & Post-operative refraction & Spherical equivalent \\
\hline 1 & 26 & 10 & $+3.50 /-1.75 \times 105$ & 2.63 \\
2 & 25 & 20 & $1 / 5 \times 70$ & -1.50 \\
3 & 25 & 15 & $+2.0 /-1.25 \times 90$ & 1.38 \\
4 & 23 & 10 & $-0.5 /-1.0 \times 60$ & $6 / 6$ \\
\hline
\end{tabular}

Patient 2 had an amblyopic eye and post-operative refraction was ambiguous and unreliable. No refraction was prescribed. The average spherical equivalent was $0.38 \mathrm{D}$, range $4.13 \mathrm{D}$. The absolute average spherical equivalent was $1.63 \mathrm{D}$. 
Table 4. Predicted refraction of biometry formulae

\begin{tabular}{ccccc}
\hline Patient no. & IOL & SRK/T & Holladay & Hoffer Q \\
\hline 1 & 36 & 0.26 & 0.89 & 1.66 \\
2 & 45 & -3.91 & -3.34 & 0.83 \\
3 & 40 & -4.03 & 3.60 & -2.58 \\
4 & 33 & -1.41 & -0.91 & -0.48 \\
\hline
\end{tabular}

Table 5. Prediction error of biometry formulae

\begin{tabular}{cccc}
\hline Patient no. & SRK/T & Holladay & Hoffer Q \\
\hline 1 & 2.37 & 1.74 & 0.97 \\
2 & 2.41 & 1.84 & -2.33 \\
3 & 5.41 & 4.98 & 3.96 \\
4 & 0.41 & -0.09 & -0.52 \\
Absolute average & 2.65 & 1.73 & 1.94 \\
\hline
\end{tabular}

relevant that accurate refraction can be difficult in the presence of neurological and structural pathology, or in the presence of amblyopia.

Refractive surprises occur because the AC depth prediction formulae have inherent restrictions. For the Hoffer Q formula, prediction of AC depth has a lower limit of $18.5 \mathrm{~mm}$, so for eyes with axial lengths less than $18.5 \mathrm{~mm}$, the post-operative AC depth prediction is the same as that for an $18.5 \mathrm{~mm}$ eye. The SRK/T AC depth prediction calculation relies on an offset (calculated using a constant of $3.336 \mathrm{~mm}$ subtracted from the ACD constant of the lens) added to the corneal dome height calculation. The effect of using a constant is greater in the short eye than the long eye and this is one reason why the SRK/T is less accurate in the short eye. Also, the retinal thickness correction factor lengthens the short and intermediate length eye: for example, a $20 \mathrm{~mm}$ eye is lengthened by $0.25 \mathrm{~mm}$, whilst the very long eye undergoes a slight shortening.

This series did show reasonable post-operative refractive errors but results would have been improved if astigmatic correction had also been attempted, and personalised constants were used. With personalised constants, the standard deviation of refractive outcomes should be tighter; ${ }^{12}$ however, the range may still be the same as without personalised constants. The relatively high prediction errors of the three formulae in short eyes has been reported previously, ${ }^{5}$ and is comparable with our findings. Prediction error is defined as the difference between the post-operative refraction predicted by the theoretical formula for the lens used versus the actual post-operative refraction. The theoretical formulae are based on the assumption of a single lens implant, so that the results must be viewed with caution. In our series, the prediction error was greatest with the SRK/T and least with the Holladay formula. This is in agreement with other studies. ${ }^{5,6}$

We did not encounter technical problems with keratometric measurement in our patient with Fuchs' corneal dystrophy, although no controls were available. Post-operatively the DLIs had no adverse effect on the cornea and it remained clear. However, all formulae showed a relatively low prediction error with this patient, which was a surprise. This could be explained by either incorrect estimation of net corneal power by keratometry, inaccurate biometry ${ }^{11}$ or a shift in lens position post-operatively. When the lens constant is back-calculated using the post-operative refraction, Surgeon Factor remained relatively unchanged at 1.75, and ACD constant 5.76 indicating the lens shifted slightly more posteriorly than predicted for a single lens. It is known that cataractous patients with Fuchs' dystrophy tend to have shallower AC depths and shorter eyes than other cataractous patients. ${ }^{13}$ As the AC depth was $2.06 \mathrm{~mm}$ by ultrasound, it is probable that the myopic surprise in this case was an error in AC depth prediction due to an unexpectedly shallow AC for the axial length. The effect of Fuchs' dystrophy on corneal power is unknown; however keratometry readings in Fuchs'

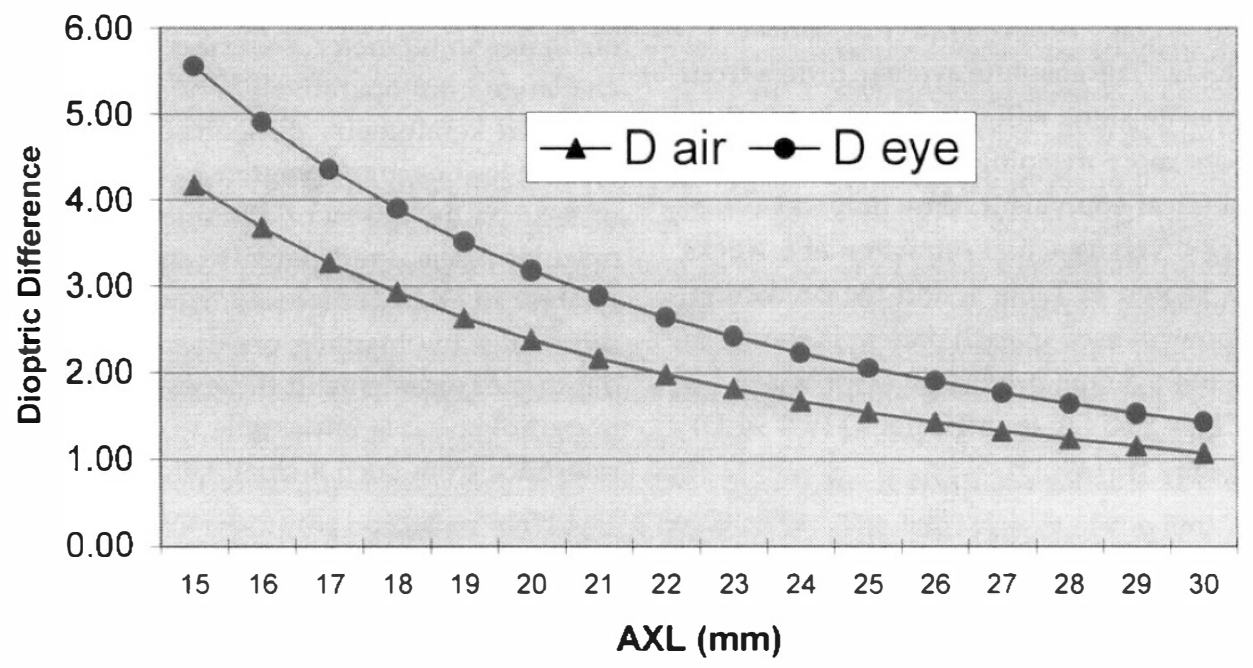

Fig. 1. The variation in vergence (measured as the dioptric distance) for each millimetre of axial length (AXL) both within the eye and in air. Note that due to reduced vergence, the dioptric distance is greater in the eye, due to the higher refractive index of 1.333 compared with air. At lengths less than $20 \mathrm{~mm}$, a $1 \mathrm{~mm}$ shift in axial length will produce at least a $3.5 \mathrm{D}$ shift in vergence within the eye. If an intraocular lens was positioned at around $4 \mathrm{~mm}$ anterior chamber depth in the same $20 \mathrm{~mm}$ eye (equivalent to an effective axial length of $16 \mathrm{~mm}$ ), a $1 \mathrm{~mm}$ difference in axial length can produce a surprise of as much as $5 \mathrm{D}$. Also note that for the emmetropic eye with an axial length of $23.65 \mathrm{~mm}$, a $1 \mathrm{~mm}$ difference produces $2.5 \mathrm{D}$ optical difference, which is exactly the same as the SRK-II regression formula for the average eye. 


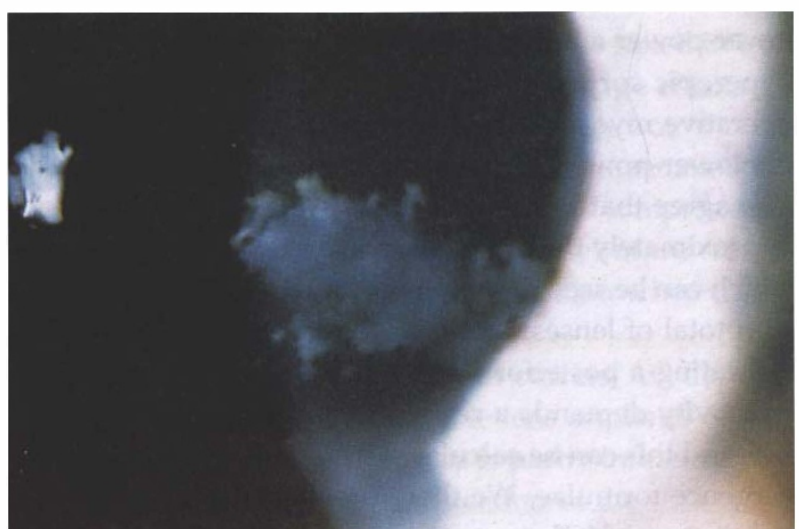

Fig. 2. Patient 1. Slit-lamp photograph of the cornea showing Saltzman-like changes of Grayson-Wilbrandt dystrophy bordering the centre of the cornea after phototherapeutic keratectomy.

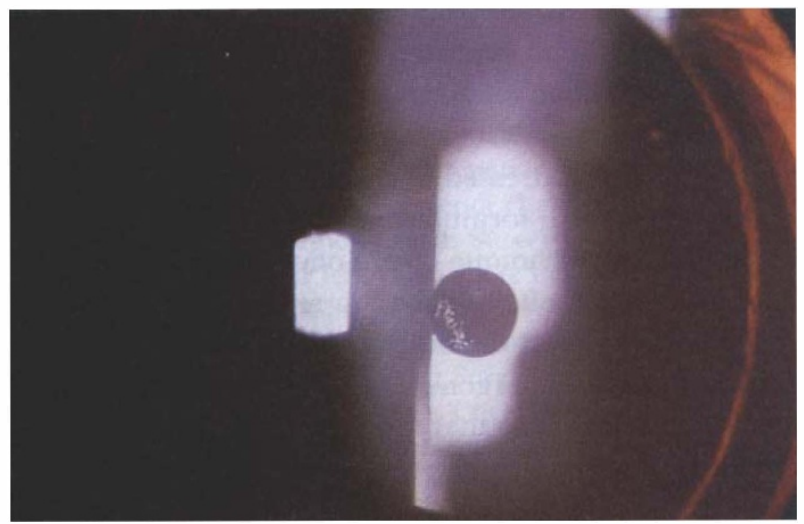

Fig. 3. Patient 1. High-magnification slit-lamp retroillumination photograph showing the point of compression of the double lens implant. Note the distance to the edge of the optic.

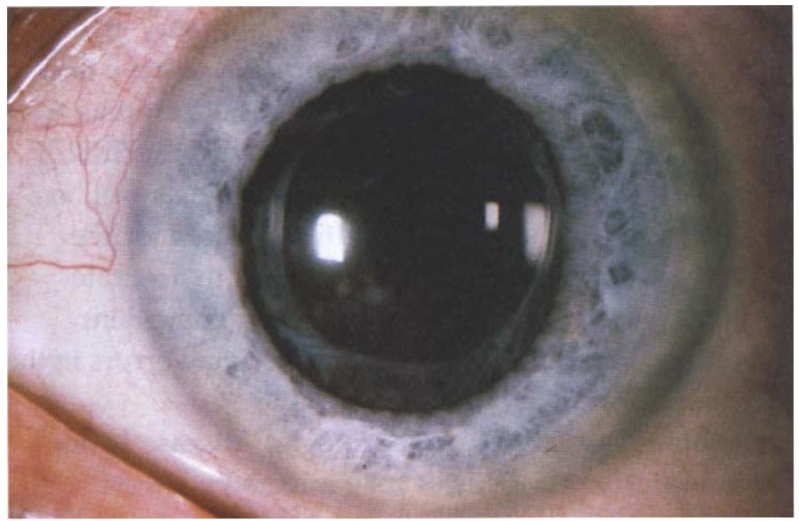

Fig. 4. Patient 1. Diffuse illumination photograph showing mild anterior capsular fibrosis over the optic only. There was no significant fibrosis that could account for mechanical compression.

dystrophy patients with cataract do not differ from those in other cataractous patients. ${ }^{13}$ In theory, any subclinical swelling of the cornea will reduce the posterior corneal power, and so it may also be possible that Fuchs' dystrophy has an effect on the estimation of corneal power, which also worked in this patient's favour to eliminate a hyperopic surprise.

Keratometric measurement problems existed in our patient with Grayson-Wilbrandt dystrophy who had also undergone previous phototherapeutic keratectomy of approximately $10 \mu \mathrm{m}$ to smooth the corneal irregularity (Fig. 2). Pre-operative hard contact lens refraction ${ }^{14,15}$ to determine corneal power was calculated at $43.66 \mathrm{D}$. The difference compared with standard keratometry was less than $0.25 \mathrm{D}$ for each of the formulae used. Therefore, the effects of the previous laser surgery and the dystrophy on corneal power in this patient were insignificant. However, the optical problems created by the inhomogeneous anterior surface of the cornea remained post-operatively, and these were overcome following rigid gas-pemeable contact lens refraction. The patient experienced an improvement in both visual acuity and subjective quality of vision during contact lens refraction achieving 6/6 acuity. The patient stated that this was the best vision he had 'ever had in his life'. Post-operative examination, however, revealed a central area of silicone intraocular lens (IOL) compression (Fig. 3) encompassing the visual axis at the point of contact of the two IOLs. The IOLs were perfectly aligned (Fig. 4) and the point of compression appeared at the apex of the curvature of both IOLs. It was also noted that the capsular bag did not show excessive fibrosis (Fig. 4). Compression of the IOL optic can result in a reduction in net optical power as well as aberrations at the margin of compression, and the reduction in net optical power could potentially be a factor contributing to the hyperopic surprise in this case. When the lens constant is back-calculated using the post-operative refraction, Surgeon Factor changes to 2.45, and ACD constant to 6.49 , indicating the lens shifted posteriorly. This is consistent with the effectivity of the double lens implant. As effectivity of the posterior IOL was not considered pre-operatively, this is likely to be the main cause for this patient's hyperopic surprise. As the quality of vision improved dramatically with rigid gas-permeable lens refraction, the edge effect of IOL central compression is therefore much less significant than the surface disease contributing to this patient's symptoms, although he did note an increase in glare under dim illumination conditions.

Why did double lens compression occur in one patient and not others? The rigidity of the PMMA lens in patient 2 accounts for the absence of lens compression but it was not seen in the other 2 patients with silicone IOLs despite careful examination. The reason for this is elusive at present. Certainly, higher power silicone IOLs are thicker centrally and the potential for compression exists. A 15D C11UB injectable equiconvex silicone lens is $1.1 \mathrm{~mm}$ thick centrally, whereas a $26 \mathrm{D}$ lens is $1.48 \mathrm{~mm}$ thick centrally. The effect of capsular bag compression may be of some importance, although as minimal fibrosis existed and the overall lens size was small $(10.5 \mathrm{~mm})$, it is unlikely that capsular compression was a significant factor. Instead it is possible that optic compression relates to the physical relationship of the curvature of the optic of both these soft lenses. One lens had a fairly steep central curvature lying adjacent to a flat curvature lens. This is analogous to the high-power lens being pressed against a flat surface. Theoretically, a force directed at the centre of a rigid arch is distributed across the arch, with 
expansion at the base, but as the silicone lenses are relatively soft, central compression may result. It is therefore the authors' recommendation that a more even mix of powers be utilised for silicone lenses if area of contact is to be minimalised.

The patient with vitelliform dystrophy showed preoperative variability in axial length measurement by contact ultrasound biometry. The range was from $18.6 \mathrm{~mm}$ to $19.27 \mathrm{~mm}$ and this difference translates to approximately $3.5 \mathrm{D}$, which agrees roughly with the prediction error. The most accurate axial length ultrasound measurement taken was used for this calculation and our most experienced medical biometry technician documented difficulty during biometry. We decided against averaging results of several measurements as this could simply cause a large spread of error. Had the axial length of $18.6 \mathrm{~mm}$ been utilised, the predicted lens powers would have been SRK/T 38.0 D, Holladay 38.7 D, and Hoffer Q 40.56 D. The Hoffer Q formula would still have resulted in the least post-operative prediction error for this patient. When the lens constant is back-calculated using the post-operative refraction, Surgeon Factor becomes 3.4, and ACD constant 7.34, indicating a much greater posterior shift in lens placement than predicted. These figures are consistent with the macula being elevated up to $1.0 \mathrm{~mm}$. The height of the macula contributed to this patient's hyperopic surprise, and our measurement preoperatively could possibly have been improved with the use of B-scan ultrasound control ${ }^{16}$ to accurately localise the height of the macula.

The problems encountered in our patient with nystagmus and poor fixation included the measurement of both axial length and keratometry, although the latter presented less of a problem due to the Wollaston prism in the keratometer stabilising ocular movements. This patient had suffered previous acute angle glaucoma and had also undergone a trabeculectomy. In retrospect, measurements may have been improved with B-scan ultrasound mode, possibly with orbital local anaesthesia. In light of the severe amblyopia, it is unlikely that an improvement in purely the refractive outcome would have produced any improvement in the unaided vision or quality of corrected vision. The finding of $5 \mathrm{D}$ of cylinder did not agree with post-operative keratometry, although tight sutures were used at the end of the procedure. The patient departed for her overseas home 3 weeks before final measurements after suture removal could be taken. As refraction was very difficult, the accuracy of the early post-operative refraction is questionable, but some of this cylinder may also have resulted from an underlying hypoplastic macula. Autorefraction was impossible due to the underlying nystagmus and both IOLs appeared centred.

Whilst we agree with the recommendation of Holladay et al..$^{5}$ that the higher implant power lens is placed posteriorly, and the lower implant power lens is placed anteriorly in the capsular bag, is it strictly necessary for the higher-power lens to lie posteriorly? A major reason for this is the ease of replacement of the lower-power anterior lens in the case of a post-operative hyperopic surprise. To assist with cases of a postoperative myopic surprise, we would recommend that the lower-power anterior lens be greater than $10 \mathrm{D}$. We also agree that the anterior lens is likely to lie in approximately the same position as a single lens implant, which can be seen with patient 1 , where despite using the sum total of lenses, there was clearly reduced effectivity indicating a posterior shift of the lens. Therefore effectivity demands a relatively higher-power posterior IOL and this can be calculated by simple geometric optics vergence formulae. We therefore recommend that parameters of IOL manufacture, especially lens thickness and the locations of the principal planes, be made available to surgeons so that appropriate vergence calculations can be made. Without this information, it is impossible for the surgeon to calculate accurately the required refractive correction and this could directly lead to a post-operative refractive surprise.

There are many issues associated with DLIs which remain poorly addressed, including the application of current theoretical formulae and the use of personalised constants. The technique of pre-operative lens determination is often unclear in reports on DLIs, and many papers report the outcomes of DLIs in eyes which appear to have undergone lens exchange for refractive surprises. We believe that more realistic reporting of outcomes should include results prior to lens exchange.

\section{Conclusions}

We report the use of double lens implant for the treatment of cataract and refractive errors in the very short eye with coincident pathology. Biometry errors which could be attributed to ocular disease have been identified. The accuracy using theoretical formulae was greatest using the Holladay and least with the SRK/T formula. Adverse findings were hyperopic as well as myopic refractive surprises. Multiple causes for the refractive surprises included difficulties in accurate biometric assessment in the presence of coincident pathology, IOL compression and underlying errors in the theoretical formulae.

Although structural or neurological disease is not a contraindication to DLIs, it may adversely affect refractive outcome. However, we have found that the range of refractive outcomes in these patients with short eyes and coincident ocular disease is comparable to published reports in non diseased eyes. The refractive effect of the coincident pathology is likely to be outweighed by standard biometry errors present in all short eyes using current theoretical formulae. The effect of Fuchs' dystrophy on keratometric estimation of corneal power is unknown and requires further investigation. Errors in corneal power estimation can be overcome using the contact lens method as is currently recommended for the patient who has undergone phototherapeutic keratectomy. Eyes with structural disease such as maculopathy warrant the assistance of Bscan ultrasound pre-operatively. 
Central compression of the IOL optic was noted in 1 of 3 patients with silicone DLIs. Glare from edge effects from central silicone lens optic compression was insignificant in our patient. Until further information is available, the visual significance from lens compression using silicone IOLs is likely to be minimal, but we would recommend a more even power distribution when using silicone foldable IOLs.

It is our opinion that the error of current AC depth prediction algorithms using double lens implants differs from single lens implants and this is partly influenced by lens combinations. Further clarification of the relationship is required. The problem of personalising constants for double lens implants remains, and we have not been able to make any recommendations arising from this series.

\section{References}

1. Freeman MH. Aberrations and ray tracing. In: Freeman MH. Optics. Oxford: Butterworth Heinmann, 1990:230-69.

2. Gayton JL. Implanting two posterior chamber intraocular lenses in microphthalmos. Ocular Surgery News 1994:64-5.

3. Hoffer KJ. Intraocular lens calculation: the problem of the short eye. Ophthalmic Surg 1981;12:269-72.

4. Hoffer KJ. Ultrasound velocities for axial eye length measurement. J Cataract Refract Surg 1994;20:554-62.

5. Holladay JT, Gills JP, Leidlein J, Cherchis M. Achieving emmetropia in extremely short eyes with two piggyback posterior chamber intraocular lenses. Ophthalmology 1996;103:1118-23.
6. Shugar JK, Lewis C, Lee A. Implantation of multiple foldable acrylic posterior chamber lenses in the capsular bag for high hyperopia. J Cataract Refract Surg 1996;22:1368-72.

7. Masket S. Piggyback intraocular lens implantation. J Cataract Refract Surg 1998;24:569-70.

8. Hoffer KJ. The Hoffer $Q$ formula: a comparison of theoretic and regression formulae. J Cataract Refract Surg 1993;19:700-21.

9. Holladay JT, Prager TC, Chandler TY, Musgrove KH, Lewis JY, Ruiz RS. A three part system for refining intraocular lens power calculations. J Cataract Refract Surg 1988;14:17-24.

10. Retzlaff JA, Sanders DR, Kraff MC. Development of the SRK/T intraocular lens implant power calculation formula. J Cataract Refract Surg 1990;16:333-40.

11. Olsen T. Sources of error in intraocular lens power calculation. J Cataract Refract Surg 1992;18:125-9.

12. Fenzl RE, Gills JP, Cherchio M. Refractive and visual outcome of hyperopic cataract cases operated on before and after implementation of the Holladay II formula. Ophthalmology 1998;105:1759-64.

13. Pitts JF, Jay JL. The association of Fuchs's corneal endothelial dystrophy with axial hypermetropia, shallow anterior chamber, and angle closure glaucoma. Br J Ophthalmol 1990;74:601-4.

14. Holladay JT. IOL calculations following radial keratotomy surgery. Refract Corneal Surg 1989;5:36A.

15. Hoffer K. Intraocular lens power calculation for eyes after refractive keratotomy. J Refract Surg 1995;11:490-3.

16. Berges O, Peuch M, Assouline M, Leteneur L, GastelluEtchegorry M. B mode guided vector A mode versus A mode biometry to determine axial length and intraocular lens power. J Cataract Refract Surg 1998;24:529-35. 